\title{
Imaging spontaneous currents in superconducting arrays of $\pi$-junctions
}

\author{
SERGEY M. FROLOV ${ }^{1}$, MICAH J. A. STOUTIMORE ${ }^{1}$, TREVIS A. CRANE ${ }^{1}$, DALE J. VAN HARLINGEN ${ }^{1 *}$, \\ VLADIMIR A. OBOZNOV², VALERY V. RYAZANOV², ADELE RUOSI ${ }^{3}$, CARMINE GRANATA ${ }^{4}$ \\ AND MAURIZIO RUSSO 4
}

\author{
${ }^{1}$ Department of Physics, University of Illinois at Urbana-Champaign, 1110 W. Green St., Urbana, Illinois 61801, USA \\ ${ }^{2}$ Institute of Solid State Physics, Russian Academy of Sciences, Chernogolovka, 142432, Russia \\ ${ }^{3}$ CNR-INFM Coherentia and Dipartimento di Scienze Fisiche, Università di Napoli, Federico II, P. Tecchio 80, 80125 Napoli, Italy \\ ${ }^{4}$ Istituto di Cibernetica 'E. Caianiello' del Consiglio Nazionale delle Ricerche, I-80078 Pozzuoli, Napoli, Italy \\ *e-mail: dvh@uiuc.edu
}

A charge current can flow between two superconductors separated by a thin barrier. This phenomenon is the Josephson effect, which enables a current to tunnel at zero voltage ${ }^{1}$, typically with no phase shift between the superconductors in the lowest-energy state. Recently, Josephson junctions with ground-state phase shifts of $\pi$, proposed by theory three decades ago $^{2}$, have been demonstrated ${ }^{3-5}$. In superconducting loops, $\pi$-junctions cause spontaneous circulation of persistent currents in zero magnetic field ${ }^{2}$, in analogy to spin-1/2 systems ${ }^{6}$. Here we use a scanning superconducting quantum interference device microscope ${ }^{7}$ to image the spontaneous zero-field currents in superconducting networks of temperature-controlled $\pi$-junctions with weakly ferromagnetic barriers ${ }^{3}$. We find an onset of spontaneous supercurrents at the $0-\pi$ transition temperature of the junctions, $T_{\pi} \approx 3 \mathrm{~K}$. We image the currents in non-uniformly frustrated arrays consisting of cells with even and odd numbers of $\pi$-junctions. Such arrays are attractive model systems for studying the exotic phases of the two-dimensional XY-model ${ }^{8,9}$ and achieving scalable adiabatic quantum computers ${ }^{10}$.

A conventional Josephson junction has the same phase in both superconductors in the ground-state wavefunction and supports a supercurrent in the direction of the applied phase difference $\phi$, as described by the classic Josephson current-phase relation $I_{\mathrm{s}}(\phi)=I_{\mathrm{c}} \sin (\phi)$, where $I_{\mathrm{c}}>0$ is the critical current. In a $\pi$-Josephson junction, it is energetically favourable for the superconducting ground-state wavefunction to change its sign across the tunnelling barrier so that a small phase difference drives a supercurrent in the opposite direction. Thus, $\pi$-Josephson junctions have a negative critical current $I_{\mathrm{c}}<0$ and a current-phase relation of the form $I_{s}(\phi)=-\left|I_{c}\right| \sin (\phi)=\left|I_{c}\right| \sin (\phi+\pi)$. The Josephson coupling can be described by the overlap of the superconducting wavefunctions that decay exponentially into the barrier from the superconducting electrodes, a phenomenon known as the proximity effect. If the barrier is a ferromagnet, the exchange interaction introduces an energy splitting between the spin-up and spin-down electrons forming the Cooper pairs, leading to a finite-centre-of-mass-momentum state for the pairs ${ }^{11-13}$. This results in a damped spatial oscillation of the proximity-induced superconducting wavefunction ${ }^{14}$, which strongly modifies the Josephson effect. In fact, such oscillations can be directly observed in the dependence of the critical current of superconductor-ferromagnet-superconductor (SFS) junctions on the ferromagnet thickness ${ }^{15}$. As the barrier thickness is varied, the coupling between the wavefunctions of the left and right superconductors oscillates and changes sign, inducing a series of transitions into and out of the $\pi$-junction state. For this experiment, we prepared arrays of $\mathrm{Nb}-\mathrm{Cu}_{47} \mathrm{Ni}_{53}-\mathrm{Nb}$ junctions near the first (thinnest) $0-\pi$ transition barrier thickness, approximately $d=11 \mathrm{~nm}$ for the weak ferromagnetic alloy used. Because the ferromagnetic barrier is diffusive, the wavelength of the order parameter oscillation depends on temperature. In our junctions, the oscillation period changes by $\sim 0.1 \mathrm{~nm}$ per degree kelvin, enabling transitions between 0 -junction and $\pi$-junction states to be observed in a single junction as a function of temperature.

A remarkable manifestation of the $\pi$-state of Josephson junctions is the generation of spontaneous persistent currents in superconducting loops incorporating odd numbers of $\pi$-junctions. Such currents are called spontaneous because no applied magnetic fields or power sources are required to create or sustain them. These currents arise to satisfy the fluxoid quantization conditions in the loops in response to the $\pi$-shifts across the junctions. Because the ground state of a loop with a single $\pi$-junction is doubly degenerate with respect to the spontaneous-current direction, $\pi$-junctions have been proposed as elements for superconducting flux qubits ${ }^{10}$. Ferromagnetic $\pi$-junctions are particularly promising as qubits owing to the absence of internal decoherence ${ }^{16}$. In contrast, other $\pi$-junction implementations rely on low barrier resistances, resulting in large dissipation and dephasing. For example, $\pi$-shift circuits $^{17}$ based on high-temperature superconductors can be prone to decoherence owing to nodal quasiparticles ${ }^{18}$, whereas recently demonstrated superconductor-ferromagnet-insulatorsuperconductor tunnel junctions realized using easily scalable niobium-trilayer and aluminium-shadow-mask technologies do not introduce additional decoherence ${ }^{19}$.

We fabricated two-dimensional square arrays of SFS Josephson junctions with various geometries. In each array, some of the cells were frustrated with three $\pi$-junctions, whereas other cells had either two or four $\pi$-junctions and were therefore 
a

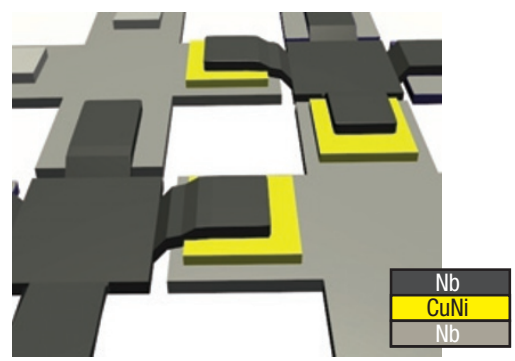

b

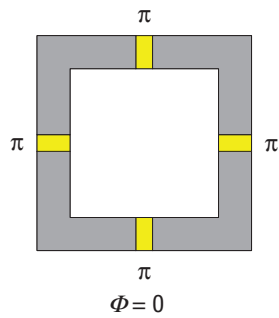

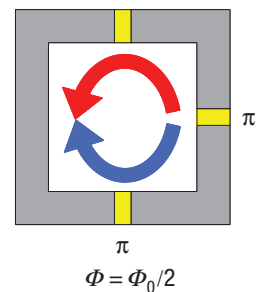

$\mathbf{G}$

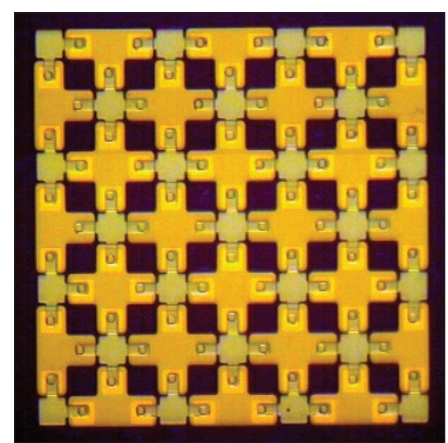

d

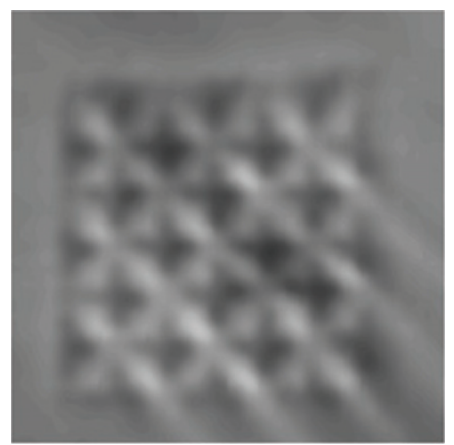

$\mathbf{e}$

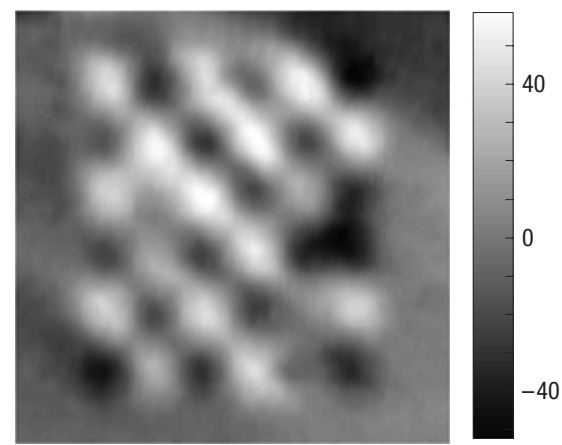

Figure $1 \pi$-junction arrays. a, Schematic diagram of a single frustrated array cell. Ferromagnetic CuNi layers windowed by a Si0 layer (not shown) are sandwiched between cross-shaped superconducting Nb electrodes to form SFS junctions. $\mathbf{b}$, In an unfrustrated cell with an even number of $\pi$-junctions, the spontaneous flux is zero in the lowest-energy state; in a frustrated cell with an odd number of $\pi$-junctions spontaneous currents generate magnetic flux of order $\pm \Phi_{0} / 2$. c, 0 tical image of a $6 \times 6$ fully frustrated array. $\mathbf{d}$, SSM image of an unfrustrated array in small applied magnetic flux ( $\ll \Phi_{0} / 2$ per cell) showing contrast from superconducting niobium. e, SSM image of a fully frustrated $6 \times 6$ array in the $\pi$-state with zero applied field. The vertical magnetic-field scale is not accurately calibrated but is approximately in units of $\mathrm{m} \Phi_{0}$ detected by the SQUID.

unfrustrated (Fig. 1b). In the unfrustrated cells, the ground-state configuration corresponds to each junction being in its lowestenergy state with a phase drop of $\pi$ and no circulating current. In contrast, frustrated cells (with sufficient inductance) require a spontaneous current to maintain flux quantization and minimize their energy. Therefore, although screening currents may circulate in all cells in the presence of applied magnetic fields, spontaneous currents appear only in frustrated cells.

An optical microscope image of a $6 \times 6$ uniformly frustrated array (all cells frustrated) is shown in Fig. 1c. To elucidate the structure of the array, a sketch of a single frustrated cell is shown in Fig. 1a. Each cell is created by overlapping two pairs of crossshaped superconducting $\mathrm{Nb}$ electrodes of thickness $100 \mathrm{~nm}$ (base) and $240 \mathrm{~nm}$ (top). An insulating layer of $150 \mathrm{~nm}$ thick SiO with windows of area $4 \times 4 \mu \mathrm{m}^{2}$ created by a lift-off lithography process separates the two superconducting layers. In three of the four overlap areas, ferromagnetic $\mathrm{Cu}_{47} \mathrm{Ni}_{53}$ barriers of thickness $d=11 \mathrm{~nm}$ are deposited to create SFS Josephson junctions. In the fourth overlap area, the base and the top superconductors are connected directly to form a superconducting contact instead of an SFS junction. Unfrustrated cells would have ferromagnetic barriers in all four (or two of four) overlap areas. The periodicity of our arrays is $30 \mu \mathrm{m}$, with each cell having an open area of $15 \times 15 \mu \mathrm{m}^{2}$, corresponding to a geometric inductance of $\sim 25 \mathrm{pH}$.

The scanning superconducting quantum interference device (SQUID) microscope (SSM) measures the average vertical magnetic field in a superconductor pickup loop scanned over the surface of a planar sample. The pickup loop, which is coupled to a d.c. SQUID detector via a superconducting flux transformer, is fabricated on a Si wafer that is bevelled to form a tip. The sensor assembly is hinged so that it rests at a small angle $\left(\sim 5^{\circ}\right)$ from the substrate with the tip in contact with the surface, maintaining the pickup coil at a distance of $2-3 \mu \mathrm{m}$ from the surface. The SSM has a spatial resolution of $5-10 \mu \mathrm{m}$, determined by the size of the pickup loop, and a magnetic flux sensitivity of $10^{-5} \Phi_{0}$, where $\Phi_{0} \approx 2.05 \times 10^{-15} \mathrm{~Wb}$ is the quantum of magnetic flux. The SSM operates in a high-permeability $\mu$-metal-shielded cryostat with a residual magnetic field of less than $10 \mathrm{mG}$ and a base temperature of $1.5 \mathrm{~K}$. A uniform magnetic field up to $100 \mathrm{G}$ perpendicular to the scanning plane can be applied from a Helmholtz coil. SSM imaging has also been used in the past to investigate spontaneous currents in loops incorporating $d$-wave superconductors ${ }^{20}$, in which intrinsic phase shifts of $\pi$ between orthogonal tunnelling directions ${ }^{21}$ cause similar phase frustration.

After the residual magnetic field in the cryostat was compensated with the magnetic field applied from a Helmholtz coil to yield net zero magnetic field, images of unfrustrated arrays showed no contrast at all temperatures. However, in a magnetic field of $1-5 \mathrm{mG}$, below the threshold field at which magneticfield-induced vortices enter the array, the array structure becomes visible because the superconducting islands screen the inductance of the SQUID pickup coil, as shown in Fig. $1 \mathrm{~d}$ for a $6 \times 6$ cell array. In contrast, all $\pi$-junction arrays with frustrated cells show spontaneous circulating currents. As an example, Fig. 1e shows an SSM image of a uniformly frustrated $6 \times 6$ array cooled below the $\pi$-state cross-over in zero magnetic field. Over most of the array, the arrangement of spontaneous currents is antiferromagnetic, with the direction of the spontaneous currents alternating in adjacent array cells to produce a checkerboard magnetic flux pattern, as expected for the ground state configuration. However, deviations 
a
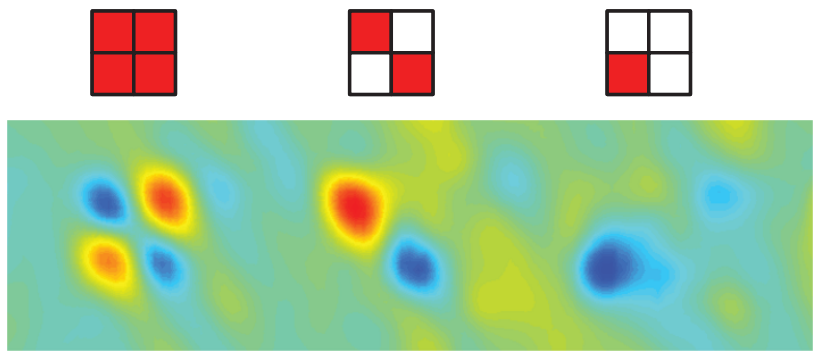

b
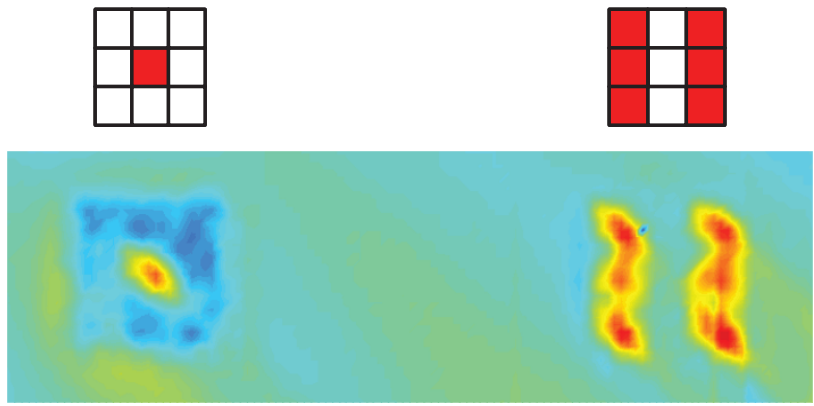

G

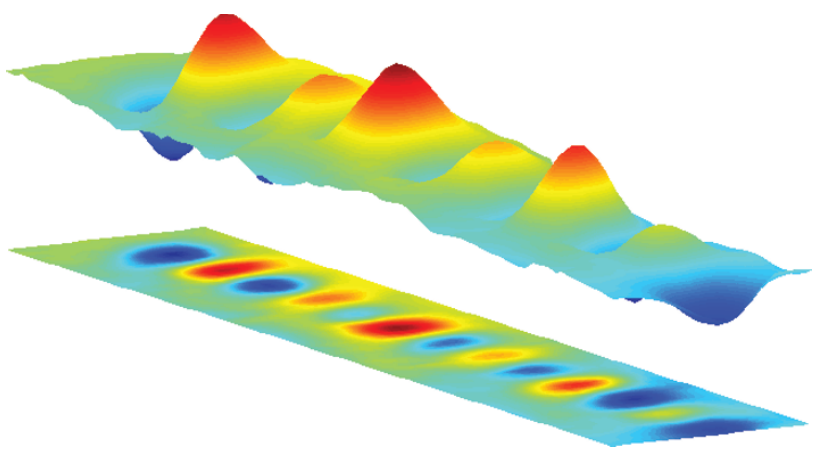

Figure 2 Designed frustration. SSM images of various array geometries in the $\pi$-state at $T=1.5 \mathrm{~K}$ with diagrams indicating the frustration patterns: $\mathbf{a}, 2 \times 2$ arrays in zero applied magnetic field showing spontaneous currents only in frustrated cells, $\mathbf{b}, 3 \times 3$ arrays in a $2 \mathrm{mG}$ applied magnetic field, c, antiferromagnetic state of a fully frustrated line array showing variations in the magnitude of the spontaneous currents.

from antiferromagnetic patterns are often observed, including in this image, because arrays can cool into metastable excited states in which one or more spontaneous currents are flipped.

Antiferromagnetic current patterns observed in a uniformly frustrated array cannot serve as definitive proof of spontaneous currents and of the existence of a $\pi$-junction state. Indeed, a magnetic-flux pattern like the one shown in Fig. 1e can occur owing to screening currents in an array of conventional junctions if the applied magnetic flux is close to $0.5 \Phi_{0}$ per cell. As a result, previous phase-sensitive experiments, although capable of demonstrating temperature-driven $0-\pi$ transitions, relied on the knowledge of the order-parameter oscillation length to define the $\pi$-junction side of the transition ${ }^{22,23}$. To distinguish unambiguously spontaneous currents induced by $\pi$-junctions from screening currents that arise in superconducting loops when magnetic fields are present, we have prepared arrays with non-uniform frustration. In Fig. 2a we show an SSM image of a variety of such arrays obtained at temperature $T=1.5 \mathrm{~K}$, along with the corresponding frustration patterns indicated by the diagrams. In all of our images taken in nominally zero applied magnetic field, currents occur only in the frustrated cells, a unique signature of the $\pi$-junction state. Figure $2 \mathrm{~b}$ shows two $3 \times 3$ arrays imaged in finite magnetic field $B \approx 2 \mathrm{mG}$. The effect of the magnetic field is to induce all of the spontaneous currents in the same direction.

Close to the transition into the $\pi$-junction state, the critical current density of SFS junctions is highly sensitive to variations in the ferromagnetic barrier thickness (at the rate of $1,000 \mathrm{~A} \mathrm{~cm}^{-2}$ per $\mathrm{nm}$ ) and to the local ferromagnetic exchange interaction. All of the frustrated cells we have imaged have the same area and contain three nominally identical SFS junctions so that the magnitudes of flux in the cells should be comparable. However, as illustrated by Fig. 2c, which shows a one-dimensional uniformly frustrated array, the magnitudes of the spontaneous currents vary from cell to cell by as much as $50 \%$ owing to a distribution of critical currents of the SFS junctions. This variation in the spontaneous currents suggests that the critical current density of our junctions has a range of $100-300 \mathrm{~A} \mathrm{~cm}^{-2}$ at $T=1.5 \mathrm{~K}$, consistent with the effective barrier-thickness variations of 1-5 $\AA$ previously observed in such junctions ${ }^{24}$.

The energies of spontaneous current configurations can be obtained from simulations of the Josephson phase dynamics of the arrays. In the simulations, the phase differences across the junctions are randomly initiated, and then allowed to evolve according to the equations describing the Josephson dynamics and the loop phase coherence until relaxed into either the ground state or into a metastable state corresponding to an irregular arrangement of spontaneous flux. We include only nearest-neighbour (adjacentcell) coupling in our simulations because longer-range magnetic coupling is negligible. The free energy can then be calculated for each state as a sum of the Josephson coupling energies of all junctions and inductive energies of the supercurrents in all loops. Calculated energy spectra (in units of the Josephson coupling energy $E_{\mathrm{J}}$ ) for $2 \times 2 \pi$-junction arrays with full frustration and checkerboard frustration (alternating frustrated and unfrustrated cells) as a function of applied magnetic field are plotted in Fig. 3. Also shown are SSM images taken at $T=1.5 \mathrm{~K}$ of the ground and metastable states in zero magnetic field. It can be noted that, in contrast to arrays of conventional 0 -junctions, the lowest-energy state is achieved at a finite value of the applied magnetic flux. When the applied magnetic flux is zero, the lowest-energy state is antiferromagnetic for both types of frustration. However, the energy gap separating the ground state from the first metastable state, with the spontaneous current of one cell reversed, is lower in checkerboard-frustrated arrays. This is because the closest current-carrying cells do not share a branch but rather couple via adjacent unfrustrated cells. It can also be seen from the simulations that the Josephson energy never reaches zero in checkerboard-frustrated arrays as it does in fully frustrated arrays. This is true for any non-uniform frustration pattern and indicates that in the $\pi$-junction state currents circulate in non-uniformly frustrated arrays at any applied magnetic field.

We can monitor the onset of spontaneous currents in an array by taking images at a series of different temperatures. We expect such currents to onset only below the $\pi$-state transition temperature $T_{\pi}$ of the individual junctions in the array. To estimate $T_{\pi}$, we measured the temperature dependence of the critical current of a single isolated SFS junction fabricated on the same chip. The critical current, obtained from the current-voltage characteristics as shown in Fig. $4 \mathrm{a}$, vanishes at $T \approx 2.9 \mathrm{~K}$, which we identify as $T_{\pi}$.

The temperature evolution of spontaneous currents in a $6 \times 6$ checkerboard-frustrated array is shown in Fig. 4b-d. No spontaneous currents were observed at temperatures well above $T_{\pi}$, as illustrated by the SSM image in Fig. 4b, which was obtained at 

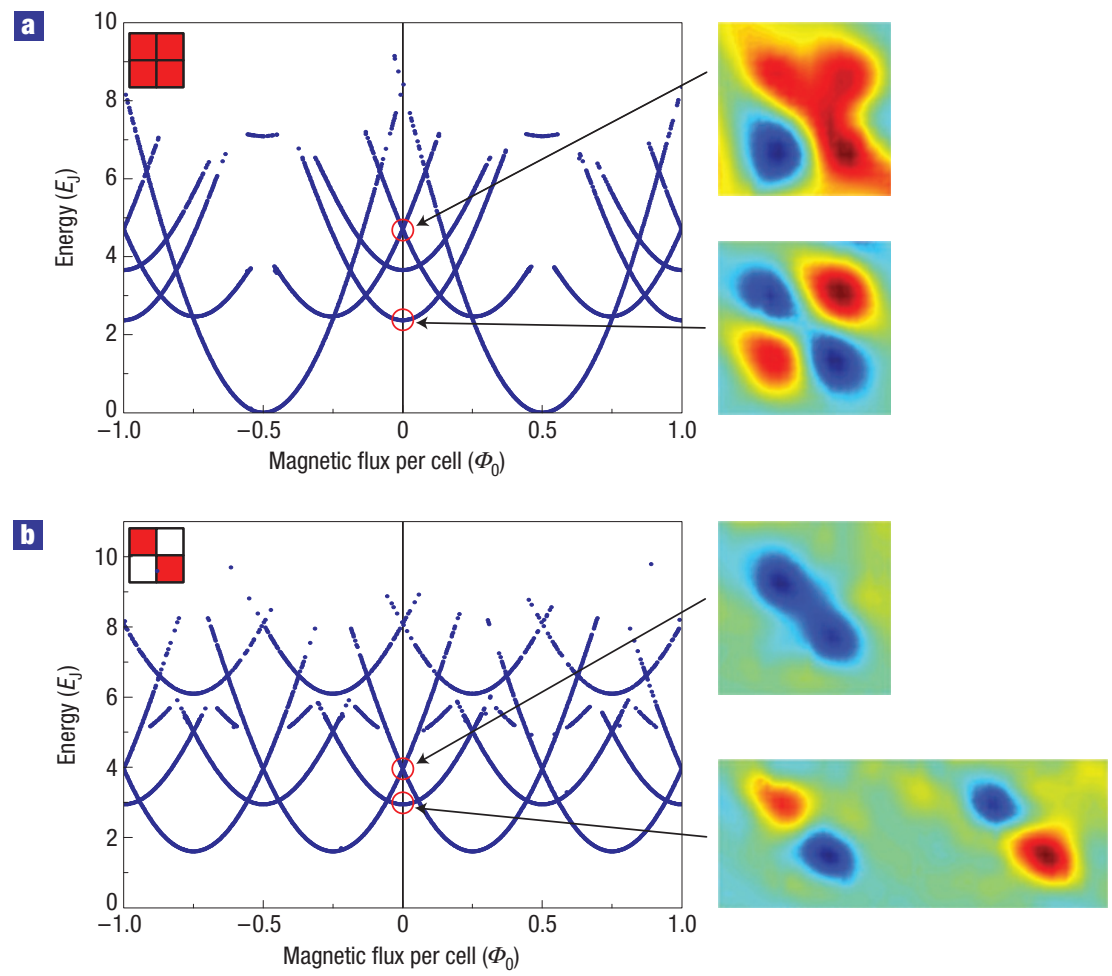

Figure 3 Spontaneous current configurations. a,b, Calculated array free energies in units of the Josephson coupling energy $E_{\mathrm{J}}=I_{\mathrm{c}} \Phi_{0} / 2 \pi$ as a function of the applied magnetic flux per cell and SSM images of corresponding spontaneous current configurations in $2 \times 2$ arrays with full frustration (a) and checkerboard frustration (b).
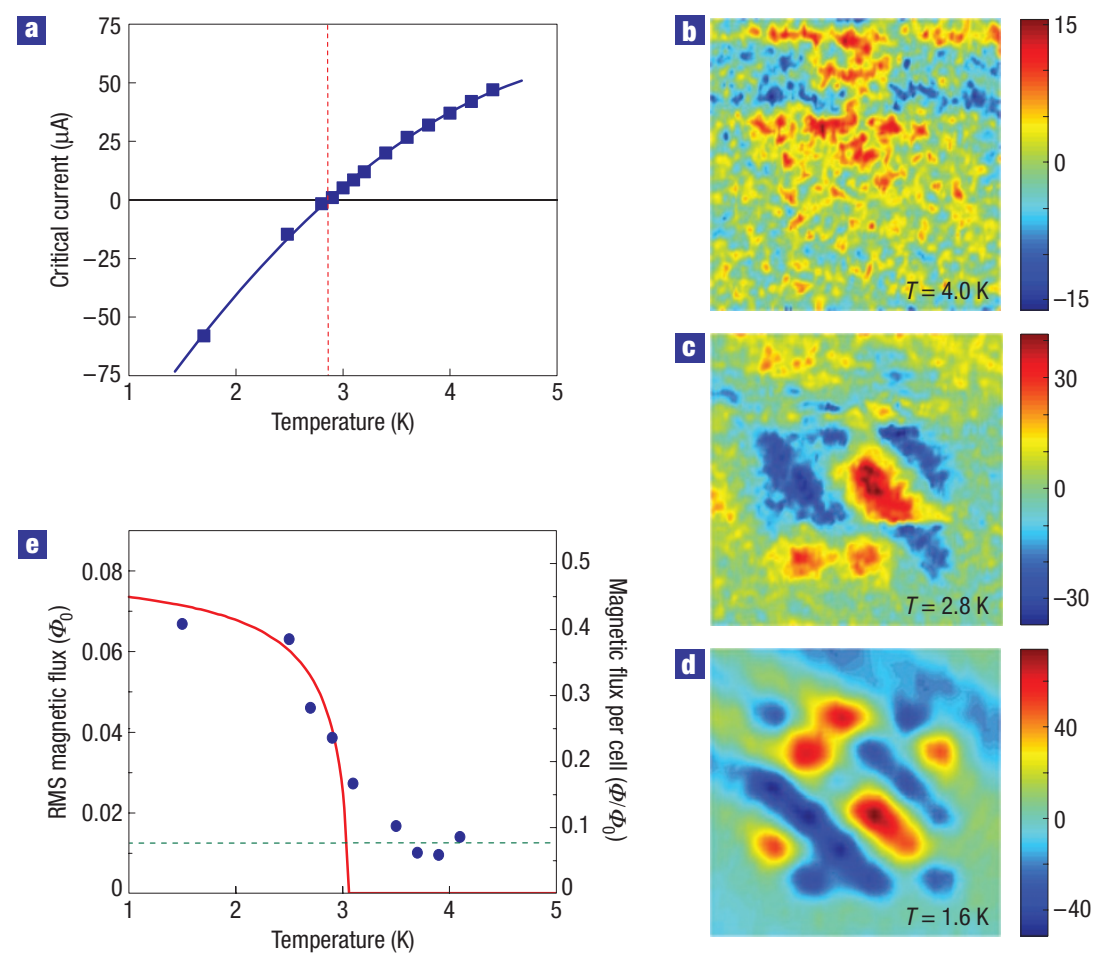

Figure 4 Temperature-driven onset of spontaneous currents. a, Temperature dependence of the critical current of a single SFS junction showing a $\pi$-junction state below $T=2.8 \mathrm{~K}$. The sign of the critical current is indicated but cannot be determined from current-voltage characteristics alone. $\mathbf{b}-\mathbf{d}$, SSM images of a $6 \times 6$

checkerboard-frustrated array taken at $T=4.0 \mathrm{~K}, 2.8 \mathrm{~K}$ and $1.6 \mathrm{~K}$. The vertical magnetic field scale is approximately in units of $\mathrm{m} \Phi_{0}$ detected by the SQUID. e, Variance of the magnetic flux generated in the array at different temperatures (solid dots) superimposed on the calculated spontaneous-flux-onset curve for a loop of inductance $L=25 \mathrm{pH}$ with a single SFS junction (solid line). The dashed line indicates the baseline set by SQUID detector noise. 
$T=4.0 \mathrm{~K}$ and shows no significant contrast. The nucleation of spontaneous currents in multiply connected circuits incorporating $\pi$-junctions depends on the energy balance between the Josephson coupling energies of the junctions $E_{\mathrm{J}}=I_{\mathrm{c}} \Phi_{0} / 2 \pi$ and the magneticfield energy in the loops $E_{\mathrm{L}}=L J^{2} / 2$ associated with the circulating current $J$. In Fig. $4 \mathrm{c}$, after we cool to $T=2.8 \mathrm{~K}$, just below the expected transition into the $\pi$-junction state, flux from spontaneous currents can be discerned but individual vortices cannot be resolved. The reason is that the critical currents of SFS junctions become very small close to $T_{\pi}$ so the ratio $E_{\mathrm{L}} / E_{\mathrm{J}} \ll 1$, making the characteristic size of the vortices much larger than a single cell. In this regime, the spontaneous flux generated by each frustrated cell is much smaller than $0.5 \Phi_{0}$ (by roughly a factor of $\left.E_{\mathrm{J}} / E_{\mathrm{L}}\right)$. Because in the vicinity of $T_{\pi}$ temperature variations of order $0.1 \mathrm{~K}-1.0 \mathrm{~K}$ result in two to three orders of magnitude change in $E_{\mathrm{J}}, \pi$-junction arrays are suitable for tunable vortex dynamics experiments, which were previously possible only at temperatures close to the superconducting critical temperature $T_{\mathrm{c}}$.

Figure $4 \mathrm{~d}$ shows an SSM image of the array obtained at $T=1.6 \mathrm{~K}$. At temperatures well below $T_{\pi}$ the spontaneous currents are bigger, and the checkerboard frustration can be resolved. In Fig. 4e we plot the r.m.s. SQUID voltage obtained by averaging the SSM signal over the array at different temperatures. There is an onset of spontaneous current that occurs at $T \approx 3 \mathrm{~K}$. For comparison, on the same graph we show the calculated temperature dependence for the onset of spontaneous magnetization in an isolated cell frustrated by a single $\pi$-junction ${ }^{2}$. The parameters of the junction were chosen to give an onset of flux near that observed in the array. We find that the onset of flux in the array is substantially broader than that for a single loop owing to variations in the SFS junction barrier thicknesses and corresponding spread in $T_{\pi}$ values of individual junctions. The ratio of the Josephson coupling energy $E_{\mathrm{J}}$ to the inductive energy $E_{\mathrm{L}}$ required to generate a magnetic flux of $0.5 \Phi_{0}$ is $E_{\mathrm{J}} / E_{\mathrm{L}}=\left(I_{\mathrm{c}} \Phi_{0} / 2 \pi\right) /\left(\Phi_{0}^{2} / 8 \pi^{2} L\right) \approx 5$ at our lowest achievable temperature $T=1.5 \mathrm{~K}$, from which we estimate that the spontaneous magnetic flux in each frustrated cell reaches $0.4 \Phi_{0}$.

Received 6 November 2006; accepted 5 October 2007; published 25 November 2007.

\section{References}

1. Josephson, B. D. Possible new effects in superconductive tunneling. Phys. Lett. 1, 251-253 (1962).

2. Bulaevskii, L. N., Kuzii, V. V. \& Sobyanin, A. A. Superconducting system with weak coupling to a current in the ground state. JETP Lett. 25, 290-294 (1977).
3. Ryazanov, V. V. et al. Coupling of two superconductors through a ferromagnet: evidence for a $\pi$-junction. Phys. Rev. Lett. 86, 2427-2430 (2001)

4. Baselmans, J. J. A., Morpurgo, A. F., van Wees, B. \& Klapwijk, T. M. Reversing the direction of supercurrent in a controllable Josephson junction. Nature 397, 43-45 (1999).

5. vam Dam, J. A., Nazarov, Y. V., Bakkers, E. P. A. M., De Franceschi, S. \& Kouwenhoven, L. P. Supercurrent reversal in quantum dots. Nature 442, 667-670 (2006).

6. Della Rocca, M. L., Aprili, M., Kontos, T., Gomez, A. \& Spathis, P. Ferromagnetic $0-\pi$ junctions as classical spins. Phys. Rev. Lett. 94, 197003 (2005).

7. Vu, L. N. \& Van Harlingen, D. J. Design and implementation of a scanning SQUID microscope. IEEE Trans. Appl. Supercond. 3, 1918-1921 (1993).

8. Korshunov, S. E. Phase transitions in two-dimensional systems with continuous degeneracy. Phys.-Uspekhi 49, 225-262 (2006).

9. Leo, C. D. \& Rotoli, G. Paramagnetic and diamagnetic states in two-dimensional Josephson-junction arrays. Phys. Rev. Lett. 89, 167001 (2002).

10. Ioffe, L. B., Geshkenbein, V. B., Feigelman, M. V., Fauchere, A. L. \& Blatter, G. Environmentally decoupled sds-wave Josephson junctions for quantum computing. Nature 398, 679-681 (1999).

11. Fulde, P. \& Ferrell, R. A. Superconductivity in a strong spin-exchange field. Phys. Rev. 135, A550-A563 (1964)

12. Larkin, A. I. \& Ovchinnikov, Y. N. Inhomogeneous state of superconductors. Sov. Phys. JETP 20, 762-769 (1965)

13. de Jong, M. J. \& Beenaker, C. W. J. Andreev reflection in ferromagnet-superconductor junctions. Phys. Rev. Lett. 74, 1657 (1995).

14. Buzdin, A. I. Proximity effects in superconductor-ferromagnet heterostructures. Rev. Mod. Phys. 77, 935-976 (2005).

15. Oboznov, V. A., Bolginov, V. V., Feofanov, A. K., Ryazanov, V. V. \& Buzdin, A. I. Thickness dependence of the Josephson ground states of superconductor-ferromagnet-superconductor junctions. Phys. Rev. Lett. 96, 197003 (2006).

16. Yamashita, T., Tanikawa, K., Takahashi, S. \& Maekawa, S. Superconducting $\pi$ qubit with ferromagnetic Josephson junction. Phys. Rev. Lett. 95, 097001 (2005).

17. Ustinov, A. V. \& Kaplunenko, V. K. Rapid single-flux quantum logic using $\pi$-shifters. J. Appl. Phys. 94, 5405-5407 (2003).

18. Fominov, Y. V., Golubov, A. A. \& Kupriyanov, M. Y. Decoherence due to nodal quasiparticles in d-wave qubits. JETP Lett. 77, 587-591 (2003).

19. Weides, M. et al. High quality ferromagnetic 0 and $\pi$ Josephson tunnel junctions. Appl. Phys. Lett. 89, 122511 (2006).

20. Hilgenkamp, H. et al. Ordering and manipulation of the magnetic moments in large-scale superconducting $\pi$-loop arrays. Nature 442, 50-53 (2003).

21. Van Harlingen, D. J. Phase-sensitive tests of the symmetry of the pairing state in the high-temperature superconductors-Evidence for $d_{x^{2}-y^{2}}$ symmetry. Rev. Mod. Phys. 67, 515-535 (1995).

22. Frolov, S. M., Van Harlingen, D. J., Oboznov, V. A., Bolginov, V. V. \& Ryazanov, V. V. Measurement of the current-phase relation of superconductor/ferromagnet/superconductor $\pi$ Josephson junctions. Phys. Rev. B 70, 144505 (2004)

23. Ryazanov, V. V., Oboznov, V. A., Veretennikov, A. V. \& Rusanov, A. Y. Intrinsically frustrated superconducting array of superconductor-ferromagnet-superconductor $\pi$ junctions. Phys. Rev. B 65 , 020501 (2001).

24. Frolov, S. M., Van Harlingen, D. J., Bolginov, V. V., Oboznov, V. A. \& Ryazanov, V. V. Josephson interferometry and Shapiro step measurements of superconductor-ferromagnet-superconductor $0-\pi$ junctions. Phys. Rev. B 74, 020503 (2006).

\section{Acknowledgements}

Research on $\pi$-Josephson junctions was supported by the National Science Foundation grant EIA-01-21568, by the US Civilian Research and Development Foundation (CRDF) grant RUP1-2691-CG-05 and by the Russian Foundation for Basic Research. The SSM imaging was supported by the Department of Energy Office of Basic Energy Sciences (DOE-BES) grant DEFG02-91-ER45439 through the Frederick Seitz Materials Research Laboratory at the University of Illinois at Urbana-Champaign. Measurements, simulations and analysis were carried out by the Urbana group; arrays were fabricated by the Chernogolovka group; d.c. SQUID detectors were fabricated by the Naples group.

Correspondence and requests for materials should be addressed to D.J.V.H.

Reprints and permission information is available online at http://npg.nature.com/reprintsandpermissions/ 